Chapter 5

\title{
Predictors of 50 Day In-Hospital Mortality in Decompensated Cirrhosis Patients with Spontaneous Bacterial Peritonitis
}

\author{
Chinmaya Kumar Bal, Ripu Daman and \\ Vikram Bhatia \\ Additional information is available at the end of the chapter \\ http://dx.doi.org/10.5772/intechopen.76795
}

\begin{abstract}
Aim: Predictors of 50 day in-hospital mortality in decompensated cirrhosis patients with spontaneous bacterial peritonitis (SBP).

Methods: 218 SBP patients admitted to intensive care units in a tertiary care hospital were retrospectively analyzed. Student $t$ test, multivariate logistic regression, Cox proportional hazard ratio, receiver operating characteristics curves and Kaplan-Meier survival analysis were utilized for statistical analysis. Predictive powers of the statistical significant variables were compared using the area under receiver operating characteristics curve (AUC). $p$ values $<0.05$ were considered statistical significant.
\end{abstract}

Results: SBP related in-hospital mortality rate was $43 \%$. Multivariate regression analysis showed acute kidney injury, hepatic encephalopathy, positive ascitic culture, leucocyte count, bilirubin, serum glutamic oxaloacetic transaminase (SGOT), Child Pugh score, and Model for End stage Liver Disease Sodium (MELD-Na) were significantly associated with 50 day in-hospital mortality. The prognostic accuracy for acute kidney injury, MELD-Na and septic shock was 77, 74 and 71\% respectively.

Conclusion: Acute kidney injury, MELD-Na and septic shock were predictors of 50 day in-hospital mortality in decompensated cirrhotic patients with SBP.

Keywords: spontaneous bacterial peritonitis, cirrhosis, acute kidney injury, model for end stage liver disease sodium 


\section{Introduction}

SBP is defined as an ascitic fluid infection without an evident intra-abdominal surgicallytreatable source [1]. SBP is a major complication of decompensated cirrhosis with ascites [2]. The diagnosis of SBP is established based on diagnostic paracentesis with an elevated ascitic fluid absolute polymorphonuclear leucocyte (PMN) count $\left(\geq 250\right.$ cells $\left./ \mathrm{mm}^{3}\right)$ and/ or a positive ascitic fluid bacterial culture. The most common pathogens involved are Gram-negative bacteria (60\%), usually Escherichia coli or Klebsiella pneumonia [3]. In about $25 \%$ of the cases, Gram-positive bacteria are involved, mainly Streptococcus species and Enterococci [3]. The prevalence of SBP is up to $30 \%$ in hospitalized cirrhotic patients with ascites [4]. Despite intensive management, the in-hospital mortality remains between 20 and $40 \%$ [5]. MELD scores have clinical utility in terms of predictive ability in SBP patients $[6,7]$. Acute kidney injury (AKI) and septic shock are fairly common in patients with decompensated cirrhosis with ascites $[8,9]$. Hence our study aimed to have a collective approach to determine common prognostic factors predicting SBP related in-hospital mortality. We also compared the predictive powers of AKI, MELD-Na and septic shock to predict 50 day in-hospital mortality.

\section{Materials and methods}

\subsection{Patients}

Retrospective analysis and review of 218 adult patients admitted to hepatology ICU with a diagnosis of SBP was done. The study was approved by the Institutional review Board and was conducted with the provisions of Declaration of Helsinki [10].

The diagnosis of cirrhosis was based on clinical, laboratory and imaging findings. SBP was diagnosed by diagnostic paracentesis in the presence of $\mathrm{PMN} \geq 250$ cells $/ \mathrm{mm}^{3}$ in the peritoneal fluid with positive culture report and the absence of the features suggestive of secondary bacterial peritonitis [11].

Data from patient's medical records were collected and tabulated. It comprises of demographics, etiology of liver disease, severity of liver disease, laboratory parameters, co-existing medical conditions (diabetes mellitus, hepatocellular carcinoma), previous medication use, organ failure, ascitic fluid analysis results, duration of ICU stay, and patient outcome. In culturepositive cases growth of the organisms and antibiotic sensitivity were recorded.

MELD-Na score was based on laboratory parameters (bilirubin, creatinine levels and INR) collected at admission and determined by using the Internet site MELD calculator [12]. Diagnostic ascitic tapping was done upon admission to ICU in all patients with ascites except in those with severe coagulopathy. Ascitic fluid was sent for differential cell count and culture. Blood sample was also sent for culture at admission in all patients. Antibiotics administered in patients based on previous antibiotic exposure and based on culture and sensitivity. 
No patient underwent fluid restriction or hypertonic saline for management of dilution hyponatremia.

AKI was defined by AKIN (acute kidney injury network) criteria [8]. AKI was managed by intravenous vasopressors (terlipressin) and intravenous albumin infusions. The dose was titrated as-per response and tolerance. Intravenous albumin was used in all patients, with a minimal daily dose of $20 \mathrm{~g}$ and increased to up to $60 \mathrm{~g} / \mathrm{d}$ [13], titrated by clinical monitoring and hourly urine output. We did not stratify renal dysfunction into hepatorenal syndrome (HRS) and non-hepatorenal syndrome. Renal replacement therapy (RRT) was used at the bedside to correct fluid overload, ascites, and electrolyte dysfunction. We did not consider advanced liver disease as a contraindication to RRT in our patient cohort.

We defined septic shock according to the American college of chest physicians/society of critical care medicine consensus conference [14].

\subsection{Exclusion criteria}

Patients with cirrhosis and ascites fluid PMN $<250$ cells $/ \mathrm{mm}^{3}$. Patients admitted from the community with SBP. Patients presented with ascites unrelated to cirrhosis. Patients with secondary peritonitis, variceal hemorrhage, advanced malignancy and HIV.

\subsection{Statistical analysis}

All statistical analyses were conducted using Stata version 14 for windows. The continuous clinical and biochemical variables and prognostic scores were expressed as mean \pm standard deviation. All the variables were assumed to be normally distributed with equal variance. The means were compared using student's $t$ test. Categorical variables were expressed as proportions and compared with logistics regression. All significant variables were analyzed using multivariate logistics regression. Cox proportional hazard model was used to analyze the hazard ratio of the predictors adjusted by age and gender. Receiver operating characteristics (ROC) curves were plotted for prognostic variables (MELD-Na, AKI and) to measure the predictive accuracy. The best cut-off point for MELD-Na was created using the ROC analysis to determine 50 day in-hospital mortality risk. The sensitivity, specificity, positive predictive values (PPV), negative predictive values (NPV), positive and negative likelihood ratio were calculated for each predictor variable so that patients could be correctly classified for each prognostic model. For all analyses, $\mathrm{p}$ value $<0.05$ was considered statistically significant. STROBE checklist for retrospective analysis was performed.

\section{Results}

A total of 218 patients with decompensated cirrhosis with ascites and SBP were included in the study. Patients that were diagnosed with SBP for the first time were $97 \%(n=211)$ with only $0.03 \%(n=7)$ had more than one episode previously. The 50 day in-hospital mortality 


\begin{tabular}{|c|c|c|c|c|}
\hline Variables & Overall $(n=218)$ & Survivors $(n=124)$ & Deaths $(n=94)$ & P-value \\
\hline \multicolumn{5}{|l|}{ Demographic data } \\
\hline Age (yrs) mean \pm SD & $49.90 \pm 12.52$ & $49.86 \pm 13.37$ & $49.96 \pm 11.37$ & 0.950 \\
\hline Male (\%) & $177(81.19 \%)$ & $99(79.84 \%)$ & $78(82.98 \%)$ & 0.557 \\
\hline \multicolumn{5}{|l|}{ Etiology of cirrhosis (\%) } \\
\hline Ethanol & $100(45.87 \%)$ & $48(38.71 \%)$ & $52(55.32 \%)$ & 0.689 \\
\hline Crypto/NAFLD & $63(28.90 \%)$ & $38(30.65 \%)$ & $25(26.60 \%)$ & 0.104 \\
\hline $\mathrm{HCV}$ & $23(10.55 \%)$ & $16(12.905)$ & $7(7.45 \%)$ & 0.068 \\
\hline \multicolumn{5}{|l|}{ Clinical data (\%) } \\
\hline $\begin{array}{l}\text { Hepatocellular } \\
\text { carcinoma }\end{array}$ & $17(7.80 \%)$ & $9(7.26 \%)$ & $8(8.51 \%)$ & 0.733 \\
\hline Diabetes & $47(21.56 \%)$ & $27(21.77 \%)$ & $20(21.28 \%)$ & 0.929 \\
\hline Acute kidney injury & $99(45.41 \%)$ & $35(28.23 \%)$ & $64(68.09 \%)$ & $<0.001$ \\
\hline Respiratory failure & $10(4.59 \%)$ & $6(4.84 \%)$ & $4(4.26 \%)$ & 0.978 \\
\hline $\begin{array}{l}\text { Hepatic } \\
\text { encephalopathy }\end{array}$ & $109(50.0 \%)$ & $50(40.32 \%)$ & $59(62.77 \%)$ & 0.001 \\
\hline Septic shock & $28(12.84 \%)$ & $4(3.23 \%)$ & $24(25.53 \%)$ & $<0.001$ \\
\hline Positive culture & $48(22.02 \%)$ & $21(16.94 \%)$ & $27(28.72 \%)$ & 0.038 \\
\hline \multicolumn{5}{|c|}{ Laboratory data* $($ mean $\pm S D)$} \\
\hline $\begin{array}{l}\text { Ascitic neutrophil } \\
\text { count (cells } / \mathrm{mm}^{3} \text { ) }\end{array}$ & $3346.07 \pm 4700.60$ & $3899.28 \pm 5003.75$ & $2616.30 \pm 4182.81$ & 0.040 \\
\hline Hemoglobin (g/dl) & $9.42 \pm 1.88$ & $9.58 \pm 1.77$ & $9.21 \pm 2.01$ & 0.154 \\
\hline $\begin{array}{l}\text { Platelet count } \\
(\mathrm{mmol} / \mathrm{L})\end{array}$ & $128.24 \pm 102.11$ & $138.43 \pm 111.25$ & $115.03 \pm 87.69$ & 0.095 \\
\hline $\begin{array}{l}\text { Leucocyte count } \\
\left(10^{3} / \mu \mathrm{L}\right)\end{array}$ & $13.30 \pm 9.35$ & $11.86 \pm 8.65$ & $15.17 \pm 9.92$ & 0.009 \\
\hline Sodium (mEq/L) & $132.14 \pm 7.69$ & $132.50 \pm 6.54$ & $131.67 \pm 9.01$ & 0.454 \\
\hline Bilirubin (mg/dL) & $8.17 \pm 8.81$ & $5.85 \pm 6.27$ & $11.24 \pm 10.61$ & $<0.001$ \\
\hline Albumin (g/dL) & $2.32 \pm 0.50$ & $2.35 \pm-0.48$ & $2.28 \pm 0.52$ & 0.250 \\
\hline INR & $2.31 \pm 1.11$ & $2.09 \pm 1.08$ & $2.59 \pm 1.08$ & 0.001 \\
\hline AST (U/L) & $59.66 \pm 109.81$ & $79.23 \pm 98.71$ & $171.3 \pm 321.94$ & 0.003 \\
\hline ALT (U/L) & $59.66 \pm 109.81$ & $46.49 \pm 72.93$ & $77.04 \pm 143.41$ & 0.041 \\
\hline Urea (mg/dL) & $70.31 \pm 52.42$ & $62.24 \pm 48.23$ & $80.94 \pm 55.98$ & 0.008 \\
\hline Creatinine $(\mathrm{mg} / \mathrm{dL})$ & $1.67 \pm 1.29$ & $1.58 \pm 1.39$ & $1.80 \pm 1.15$ & 0.217 \\
\hline \multicolumn{5}{|l|}{ Scores $($ mean $\pm S D)$} \\
\hline CTP (B/C) & $10.72 \pm 1.82$ & $10.50 \pm 1.95$ & $11.02 \pm 1.60$ & 0.034 \\
\hline
\end{tabular}




\begin{tabular}{lllll}
\hline Variables & Overall $(\mathbf{n}=\mathbf{2 1 8})$ & Survivors $(\mathbf{n}=\mathbf{1 2 4})$ & Deaths $(\mathbf{n}=\mathbf{9 4})$ & P-value \\
\hline MELD & $24.79 \pm 8.28$ & $22.20 \pm 7.59$ & $28.20 \pm 7.94$ & $<0.001$ \\
MELD-Na & $27.53 \pm 7.57$ & $25.21 \pm 7.44$ & $30.59 \pm 6.62$ & $<0.001$ \\
\hline
\end{tabular}

*Results obtained on the day of diagnosis of SBP.

Table 1. Baseline characteristics of the hospitalized patients with spontaneous bacterial peritonitis in decompensated cirrhosis.

rate was $43.11 \%(n=94)$. Median survival duration for those who died was 9 days. In univariate analysis AKI, hepatic encephalopathy, total leucocyte count, serum bilirubin, INR, SGOT, and MELD-Na are significantly associated with in hospital mortality in patients with SBP (Table 1).

The baseline characteristics of the demographics, etiology, clinical and laboratory data shown in Table 1. Mean age was $49.90 \pm 12.52$ years and the male was predominant $(83 \%)$. Most common etiology of liver cirrhosis was ethanol induced (45.87\%) followed by crypto/ NAFLD (28.9\%). Liver cirrhosis due to HCV infection constitute only $11 \%$ in our study. Total $50.0 \%$ patients $(n=109)$ had hepatic encephalopathy with $62.77 \%$ deaths $(n=59$, $\mathrm{p}=0.001)$. Overall $45.11 \%$ subjects $(\mathrm{n}=99)$ had AKI who were hospitalized, out of which $68.09 \%(n=64, p \leq 0.001)$ died. Compared with survivors, the non-survivors had a higher proportion of septic shock ( 25.53 vs. $3.23 \%), p<0.001$. Mean leucocyte count, bilirubin, INR, AST were significantly higher in the persons who died in comparing to the survivors. Mean MELD-Na score was higher among the non-survivors compared with the survivors $(30.59 \pm 6.62$ vs. $25.21 \pm 7.44)(\mathrm{p}<0.001)$. It is surprising to notice that $\mathrm{CTP}(\mathrm{B} / \mathrm{C})$ score was not different among the survivors and non-survivors. The mean CTP scores were high $(10.72 \pm 1.82)$.

On multivariate regression analysis, AKI $(p=0.001)$, septic shock $(p=0.029)$, MELD-Na $(\mathrm{p}<0.001)$ were found to be independent predictors of 50 day in-hospital mortality in patients with SBP (Table 2). Cox proportional hazard model showed the hazard ratio of AKI was $2.16(95 \% \mathrm{CI}=1.36-3.42)$, septic shock (HR 1.73, 95\% CI =1.05-2.83) and MELD-Na (HR 1.1, $95 \% \mathrm{CI}=1.02-1.21)$. ROC curve for AKI, septic shock and MELD-Na had better prognostic

\begin{tabular}{lll}
\hline Variables & $\begin{array}{l}\text { Hazard ratio }^{*} \\
\mathbf{9 5 \%} \mathbf{C I})\end{array}$ & P value \\
\hline AKI & $2.16(1.36-3.42)$ & 0.001 \\
Septic shock & $1.73(1.05-2.83)$ & 0.029 \\
MELD-Na & $1.06(1.02-1.09)$ & $<0.001$ \\
\hline
\end{tabular}

*Hazard ratio adjusted for age and gender.

AKI, acute kidney injury; MELD-Na, model for end-stage liver disease sodium.

Table 2. Cox proportional regression analysis of risk factors for SBP related in-hospital related mortality. 


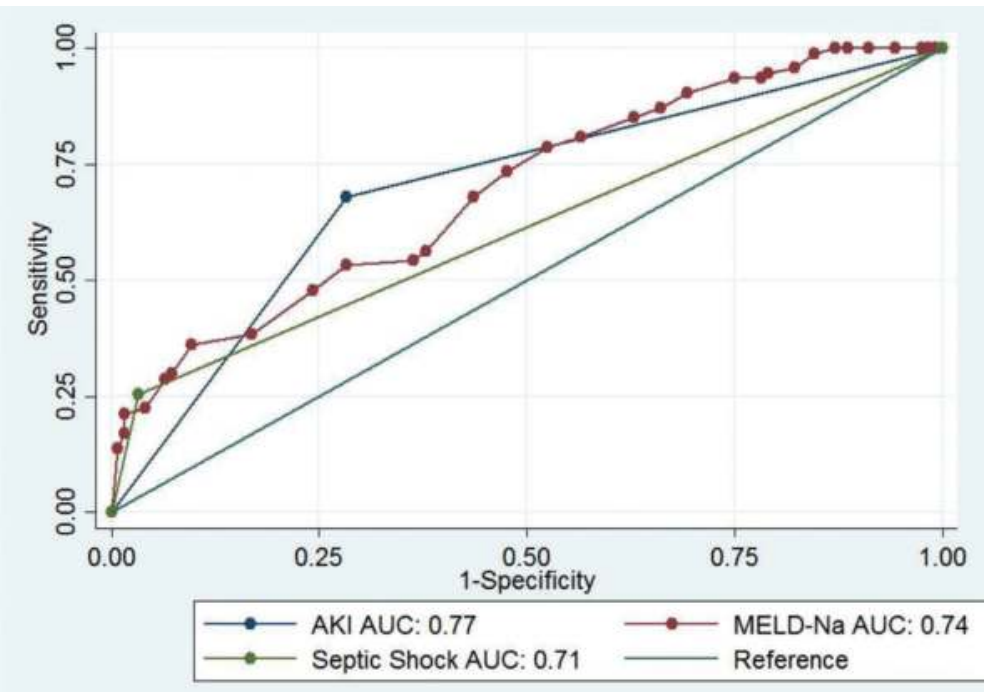

Figure 1. Receiver operator characteristic (ROC) curve for AKI, septic shock and MELD-Na had better prognostic accuracy for 50 day in-hospital mortality in patients with SBP.

\begin{tabular}{lllllll}
\hline Predictors & Sensitivity & Specificity & PPV & NPV & +LR & -LR \\
\hline AKI & 64.6 & 74.8 & 68.1 & 71.8 & 2.56 & 0.47 \\
Septic shock & 85.7 & 63.2 & 25.5 & 96.8 & 2.33 & 0.23 \\
MELD-Na(28) & 92.9 & 60.3 & 24.5 & 97.9 & 2.34 & 0.12 \\
\hline
\end{tabular}

${ }^{1}$ Cut off score for MELD-Na, PPV: positive predictive value, NPV: negative predictive value, + LR: positive likelihood ratio, -LR: negative likelihood ratio, AKI: acute kidney injury, MELD-Na: model for end stage liver disease sodium.

Table 3. Diagnostic accuracy of prognostic variables to predict SBP related in-hospital mortality.

accuracy for 50 day in-hospital mortality in patients with SBP (Figure 1). AKI had highest Area Under Curve (AUC) 0.77, 95\% confidence interval (95\% CI $=0.71-0.83)$, followed by MELD-Na (AUC0.74,95\% CI=0.69-0.79), septicshock (AUC0.71,95\% CI=0.65-0.77). Table3 reported the sensitivity, specificity, PPV, NPV, positive likelihood ratio (+LR) and negative likelihood ratio (-LR) for these predictors. The cut off for MELD-Na derived from the ROC with the best ability to predict 50 day in-hospital mortality in decompensated cirrhotic patient with SBP was 28, with sensitivity $92.9 \%$, specificity $60.3 \%$, and NPV of $97.9 \%$. The Kaplan-Meier survival analysis was plotted for the 50 day survival in SBP patients along with individual prognostic variables like AKI, MELD-Na, and septic shock (Figure 2). 

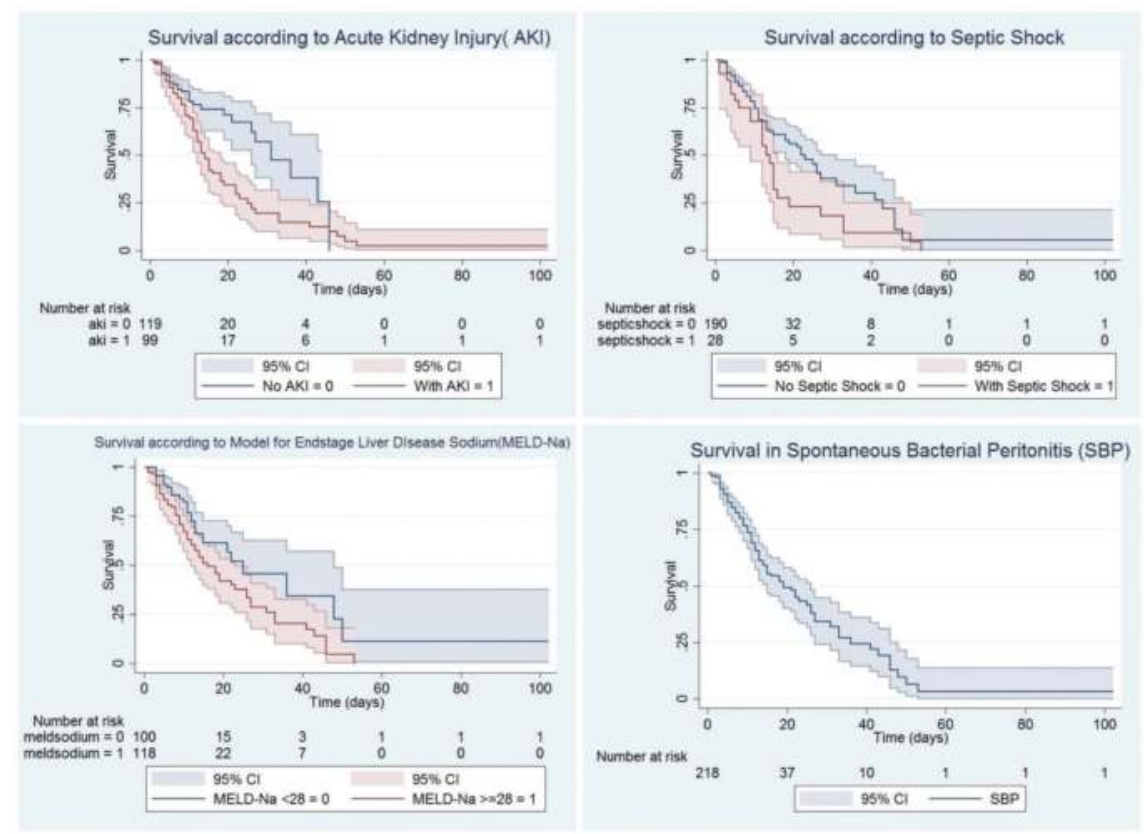

Figure 2. Kaplan-Meier survival analysis was plotted for the 50 day survival in SBP patients along with individual prognostic variables like AKI, MELD-Na, and septic shock.

\section{Discussion}

The prevalence of SBP in outpatients has been reported to be 1.5-3.5\% [15]. The prevalence among in-patients is around 10\% [16]. Half of the episodes of SBP are nosocomial related. In our retrospective observational study $43 \%(n=93)$ of the decompensated cirrhotic patients with SBP died within 50 day of hospital admission. Our study considered a variety of prognostic factors that can be used to predict mortality in hospitalized patient with SBP. Our findings support previous study results that variables like hepatic encephalopathy, total leucocyte count, serum bilirubin, SGOT, INR, Child Pugh scores are significantly associated with mortality $[5,6]$. The MELD score was found to be an independent predictor of death in cirrhotic patients [7] including those waiting for liver transplantation. MELD-Na is a better prognostic model compared with MELD model [17] for predictive accuracy of mortality in liver cirrhosis. Serum creatinine level measurement for renal dysfunction in decompensated liver cirrhosis is erroneous. It can be due to decrease in creatinine production in liver and associated muscle wasting due to malnutrition [18]. We found that AKI, MELD-Na and septic shock are significant in terms of predicting mortality. We overlooked other predictable variables like total leucocyte count, serum bilirubin, INR to avoid confusion and complexity as those were the components in our predictive model like MELD-Na and septic shock. 
Our study demonstrated that AKI has the single best predictive ability (AUC $=0.77$ ) followed by MELD-Na $(A U C=0.74)$ and septic shock $(A U C=0.71)$. We set a cut-off value for MELD-Na to be 28 . It has sensitivity $92.9 \%$ and NPV of $97.9 \%$. AKI has the highest hazards of mortality (HR 2.16, 95\% CI 1.36-3.42) followed by septic shock (HR 1.73, 95\% CI 1.05-2.83) and MELD-Na (HR 1.06, 95\% 1.02-1.09). We plotted graph of Kaplan-Meier survival analysis. The graph illustrated AKI, MELD-Na, and septic shock as predictors for the 50 day in-hospital mortality in decompensated patients with SBP. We hope that these prognostic variables can help in the further improvement of the quality of care of hospitalized SBP patients. The cut-off value 28 for MELD-Na can be utilized to stratify patients diagnosed with SBP into high risk category upon hospital admission.

Diagnosis of SBP is based on the demonstration of an absolute number of polymorphonuclear cells in ascitic fluid equal to or greater than $250 / \mathrm{mm}^{3}$ with culture positivity. There is a controversy regarding antibiotic therapy in culture positivity with normal ascitic fluid PMN count (bacteriascites). Runyon et al. recommend antibiotic treatment only if the patient shows signs of infection [2]. The first-line of choice antibiotics for treatment of SBP include third generation cephalosporins, amoxicillin-clavulanic acid, ciprofloxacin, and ofloxacin [19]. There is increasing evidence of antibiotic resistance [20].

In our study ascitic fluid culture is positive in $40 \%$ of all cases. The most common growth include Gram-negative bacteria (GNB), mostly Escherichia coli and Gram-positive cocci (mainly streptococcus species and enterococci) [3]. The epidemiology of bacterial infections differs between community-acquired (in which GNB infections predominate) and nosocomial infections (in which Gram-positive infections predominate) [3]. The infections resistant to first lines of antibiotics are usually caused by Enterococcus faecium and extended-spectrum $\beta$-lactamase (ESBL) organism like Enterobacteriaceae [21]. There are compelling evidence that nosocomial SBP should be treated with carbapenems or with tigecycline [22].

We included only nosocomial SBP patients in our study because most of the ICU admissions includes referred patients from other hospitals, with a variable but inconsistent antibiotic exposure. Only a minority of our patients are admitted directly from the community, and usually to the wards and not to the ICU. Hospital-acquired infections due to a higher incidence of multidrug resistance (third-generation cephalosporins) were an independent predictor of death [23]. These results are in keeping with recent data showing higher rates of drug resistance in patients with nosocomial SBP and increased rates of and death in patients with multidrug resistance [24].

Dr. Garcia-Tsao recently reviewed 18 studies, and reported that the most common predictors of death were renal dysfunction, lack of SBP resolution, immunosuppressive factors, and hospital-acquired SBP [24]. It identified renal dysfunction and levels of blood urea nitrogen and creatinine as the most important variables. The mortality rate among patients with renal dysfunction was $67 \%$, compared with only $11 \%$ of patients who maintained normal renal function. Renal dysfunction was defined somewhat variably in the studies, but most defined it as a creatinine level greater than $1.5 \mathrm{mg} / \mathrm{dL}$.

Our study has certain strengths and limitations. The results clearly show that AKI has greater predictive ability than septic shock and MELD-Na as far as 50 day in-hospital mortality in SBP patient is concerned. Our study did not account for the stages of ascites. We did not stratify 
our patients according to different stages of AKI as per AKIN criteria. We did not take HRS into account in our study. The standard first line antibiotics were not used in the treatment of SBP. The choice of antibiotics coverage based of culture sensitivity and previous exposure. We did not thoroughly evaluate the antibiotic resistance in SBP patients who are culture positive at the baseline. We included only nosocomial acquired SBP. Most of our patients presented with advanced decompensated liver cirrhosis at the time of SBP diagnosis as it is a tertiary care center. The advanced liver cirrhosis was assessed by lower serum albumin, high serum bilirubin and INR values. As an observational study we were unable to assess the impact of volume expansion and SBP specific therapy on patient outcome. Our study is a single center study, these findings needed to be supplemented by multicenter prospective studies.

Our study findings can guide in advanced liver cirrhosis patients that would benefit them from intensive management where liver transplant is not feasible.

\section{Author details}

Chinmaya Kumar Bal*, Ripu Daman and Vikram Bhatia

*Address all correspondence to: chinmaya.bal@gmail.com

Department of Hepatology, Institute of Liver and Biliary Science, New Delhi, India

\section{References}

[1] Rimola A, García-Tsao G, Navasa M, Piddock LJ, Planas R, Bernard B, Inadomi JM. Diagnosis treatment and prophylaxis of spontaneous bacterial peritonitis: A consensus document. International Ascites Club. Journal of Hepatology. 2000;32:142-153. DOI: 10.1016/S0168-8278(00)80201-9

[2] Such J, Runyon BA. Spontaneous bacterial peritonitis. Clinical Infectious Diseases. 1998;27(4):669-674 quiz 675-6

[3] Dever JB, Sheikh MY. Review article: Spontaneous bacterial peritonitis-bacteriology, diagnosis, treatment, risk factors and prevention. Alimentary Pharmacology \& Therapeutics. 2015;41(11):1116-1131. DOI: 10.1111/apt.13172

[4] Gunjaca I, Francetić I. Prevalence and clinical outcome of spontaneous bacterial peritonitis in hospitalized patients with liver cirrhosis: A prospective observational study in central part of Croatia. Acta Clinica Croatica. 2010;49(1):11-18

[5] Thuluvath PJ, Morss S, Thompson R. Spontaneous bacterial peritonitis in-hospital mortality, predictors of survival, and health care costs from 1988 to 1998. The American Journal of Gastroenterology. 2001;96:1232-1236. DOI: 10.1111/j.1572-0241.2001.03708.x

[6] Dănulescu RM, Stanciu C, Trifan A. Evaluation of prognostic factors in decompensated liver cirrhosis with ascites and spontaneous bacterial peritonitis. Revista MedicoChirurgicală a Societătiii de Medici şi Naturalişti din Iaşi. 2015;119(4):1018-1024 
[7] Nobre SR, Cabral JE, Gomes JJ, Leitão MC. In-hospital mortality in spontaneous bacterial peritonitis: A new predictive model. European Journal of Gastroenterology \& Hepatology. 2008;20:1176-1181. DOI: 10.1097/MEG.0b013e32830607a2

[8] de Araujo A, Alvares-da-Silva MR. Akin criteria as a predictor of mortality in cirrhotic patients after spontaneous bacterial peritonitis. Annals of Hepatology. 2014;13(3):390-395

[9] Moreau R, Hadengue A, Soupison T, Kirstetter P, Mamzer MF, Vanjak D, et al. Septic shock in patients with cirrhosis: Hemodynamic and metabolic characteristics and intensive care unit outcome. Critical Care Medicine. 1992;20(6):746-750

[10] WMA declaration of Helsinki-ethical principles for medical research involving human subjects [internet]. 2013. Available from: https://www.wma.net/what-we-do/education/ medical-ethics-manual [Accessed: 2018-03-19]

[11] Runyon BA. The evolution of ascitic fluid analysis in the diagnosis of spontaneous bacterial peritonitis. The American Journal of Gastroenterology. 2003;98(8):1675-1677

[12] MELDNa/MELD-Na Score for Liver Cirrhosis [Internet]. 2016. Available from: https:// www.mdcalc.com/meldna-meld-na-score-liver-cirrhosis [Accessed: 2018-03-19]

[13] de Mattos A. Current indicators for use of albumin in the treatment of cirrhosis. Annals of Hepatology. 2011;10(Suppl. 1):S15-S20

[14] Bone RC, Balk RA, Cerra FB, Dellinger RP, Fein AM, Knaus WA, Schein RM, Sibbald WJ. Definitions for sepsis and organ failure and guidelines for the use of innovative therapies in sepsis. The ACCP/SCCM Consensus Conference Committee. American College of Chest Physicians/Society of Critical Care Medicine. 1992. DOI: 10.1378/ chest.101.6.1644

[15] Chu CM, Chang KY, Liaw YF. Prevalence and prognostic significance of bacterascites in cirrhosis with ascites. Digestive Diseases and Sciences. 1995;40(3):561-565

[16] Terg R, Gadano A, Cartier M et al. Serum creatinine and bilirubin predict renal failure and mortality in patients with spontaneous bacterial peritonitis: A retrospective study. Liver International. 2009;29:415-19

[17] Musskopf MI, Fonseca FP, Gass J, de Mattos AZ, John JA, de Mello Brandão AB. Prognostic factors associated with in-hospital mortality in patients with spontaneous bacterial peritonitis. Annals of Hepatology. 2012;11(6):915-920

[18] MacAulay J, Thompson K, Kiberd BA, Barnes DC, Peltekian KM. Serum creatinine in patients with advanced liver disease is of limited value for identification of moderate renal dysfunction: Are the equations for estimating renal function better? Canadian Journal of Gastroenterology. 2006;20(8):521-526

[19] European Association for the Study of the Liver. EASL clinical practice guidelines on the management of ascites, spontaneous bacterial peritonitis, and hepatorenal syndrome in cirrhosis. Journal of Hepatology. 2010;53(3):397-417. DOI: 10.1016/j.jhep.2010.05.004 
[20] Cheong HS, Kang CI, Lee JA, et al. Clinical significance and outcome of nosocomial acquisition of spontaneous bacterial peritonitis in patients with liver cirrhosis. Clinical Infectious Diseases. 2009;48:1230-1236. DOI: 10.1086/597585

[21] Ariza X, Castellote J, Lora-Tamayo J, Girbau A, Salord S, Rota R, et al. Risk factors for resistance to ceftriaxone and its impact on mortality in community, healthcare and nosocomial spontaneous bacterial peritonitis. Journal of Hepatology. 2012;56:825-832. DOI: 10.1016/j.jhep.2011.11.010

[22] Fernández J, Acevedo J, Castro M, Garcia O, de Lope CR, Roca D, Pavesi M, Sola E, Moreira L, Silva A, Seva-Pereira T, Corradi F, Mensa J, Ginès P, Arroyo V. Prevalence and risk factors of infections by multiresistant bacteria in cirrhosis: A prospective study. Hepatology. 2012;55:1551-1561. DOI: 10.1002/hep.25532

[23] Umgelter A, Reindl W, Miedaner M, Schmid RM, Huber W. Failure of current antibiotic first-line regimens and mortality in hospitalized patients with spontaneous bacterial peritonitis. Infection. 2009;37:2-8. DOI: 10.1007/s15010-008-8060-9

[24] Tandon P, Garcia-Tsao G. Renal dysfunction is the most important independent predictor of mortality in cirrhotic patients with spontaneous bacterial peritonitis. Clinical Gastroenterology and Hepatology. 2011;9(3):260-265. DOI: 10.1016/j.cgh.2010.11.038 
